\title{
Implementação de algoritmo para o controle automático de sistemas de duas entradas e duas saídas - TITO
}

\author{
Implementation of algorithm for the automatic control of two input and two output \\ systems - TITO
}

\section{Caio Mariano da Silva Anastácio 1 https://orcid.org/0000-0001-8312-927X}

\section{Péricles Guedes Alves 1,2 1 https://orcid.org/0000-0003-1436-9776}

1 Departamento de Engenharia Elétrica, Centro Universitário de Volta Redonda, Volta Redonda, Brasil.

2 Instituto Federal do Rio de Janeiro, Volta Redonda, Brasil.

E-mail do autor principal: Caio Mariano caio_anastacio@hotmail.com

\section{Resumo}

Esse artigo foi desenvolvido a partir da construção de um algoritmo capaz de realizar, em poucos segundos, a sintonização de sistemas modernos multivariáveis de duas entradas e duas saídas - TITO, baseando-se em uma nova técnica de controle, garantindo um resultado eficaz ao usuário, sem que seja necessário um conhecimento avançado em sintonização de controladores. A metodologia utilizada contou com aporte teórico presente no trabalho dos pesquisadores Ziegler e Nichols, onde realizou-se uma modificação na forma de obtenção de alguns parâmetros, bem como sua aplicação em modelos multivariáveis, divergindo assim dos pesquisadores que desenvolveram sua pesquisa em torno de sistema monovariável, de uma única entrada e uma única saída, também conhecido como sistema SISO. Com o resultado obtido, é possível afirmar quanto a efetividade do algoritmo, tornando uma alternativa viável para o controle desses sistemas.

Palavras-Chave: Sistema TITO (Two Input Two Output); Controle PID (Proporcional Integral Derivativo); Controle Automático Multivariável; Matlab.

\section{Abstract}

This paper was developed from the construction of an algorithm capable of performing, in a few seconds, the tuning of modern two-input, two-output multivariable systems - based on a new control technique, guaranteeing an efficient result to the user, without the need for advanced knowledge in driver tuning. The methodology used had a theoretical contribution in the work of the researchers Ziegler and Nichols, where a change was made in the way of obtaining some parameters, as well as its application in multivariable models, thus diverging from the researchers who developed their research around the system a single input and a single output, also known as a SISO system. With the obtained result, it is possible to affirm the effectiveness of the algorithm, becoming a viable alternative for the control of these systems.

Key-words: TITO system; PID control; Multivariable Automatic Control; Matlab 


\section{Introdução}

Os processos industriais estão, progressivamente, ficando cada vez mais complexos [1]. A modernização desses processos requer um controle de natureza multivariável, devido às múltiplas entradas e saídas que são utilizadas para descrever o modelo do sistema [1]. O sistema multivariável (MIMO - MultipleInput Multiple-Output), destaca-se pela capacidade de interação entre as malhas, isto é, uma ação de controle afeta diretamente o processo como um todo $[\mathbf{1}, \mathbf{2}]$. Devido a essa característica, é imprescindível que o projetista adote medidas que garantam segurança no ajuste do controlador empregado, impedindo que leve o sistema à instabilidade $[\mathbf{1}, \mathbf{2}]$.

O controlador Proporcional Integral Derivativo, - PID, possui uma aplicação muito expressiva dentro das indústrias. Estima-se sua presença em $96 \%$ dos processos [1, 3]. Além de sua notável utilização no meio industrial, ele também é constantemente utilizado como objeto de pesquisas na área acadêmica [4]. Um dos desafios que pode ser destacado é a capacitação de usuários e engenheiros para que seja realizada uma sintonização adequada e correta nesses controladores [5]. Uma consequência que pode ser identificada pela ausência de conhecimento desses profissionais é uma sintonia pobre, tornando o sistema com baixo desempenho, o que influencia diretamente na qualidade final do produto $[1,6,7]$.

Atualmente, é possível encontrar diversas metodologias $[4,5,8,9,10,11,12,13]$, que direcionam o usuário para alcançar um ajuste satisfatório em sistemas multivariáveis. Um dos problemas é que, várias das técnicas disponíveis requerem do usuário uma bagagem de conhecimento muito sólida em controle, que vai desde múltiplas etapas, considerações em índices de desempenho, matrizes e métodos de desacoplamento sofisticados. Neste contexto, o presente artigo apresenta uma alternativa para que usuários que desejam realizar ajuste em sistemas multivariáveis de duas entradas e duas saídas, consigam, de forma rápida e com simplicidade, obter o controle com resultados que garantam estabilidade ao processo.
A implementação de uma nova metodologia é enfatizada, juntamente com a automatização por meio de um algoritmo executado no software MATLAB, que conta com uma interface gráfica intuitiva para todos usuários, seja ele novo ou avançado no campo da engenharia de controle.

\section{Controle Moderno}

Inúmeras técnicas de controle podem ser utilizadas em sistemas modernos de duas entradas e duas saídas, como o controle adaptativo, preditivo, robusto, entre outras $[\mathbf{2}, \mathbf{1 4}, \mathbf{1 5}]$. É necessário ressaltar que, as técnicas mais atuais geralmente ficam apenas no campo teórico, devido à dificuldade de implementação, pois requer do usuário muitas vezes, um nível de instrução muito específica em cálculos de matrizes, programação e modelagem [5]. De uma forma ampla, pode-se dizer que essas metodologias de controle são capazes de alterar o seu comportamento, moldando juntamente com as perturbações e dinâmica do processo envolvido [14]. Deve-se levar em conta que, para cada processo, a aplicação é definida pela singularidade do controlador de atuar nas propriedades que o usuário julgar mais importantes [1, 2]. Uma especificidade do sistema multivariável está na forma em que uma variável do sistema influencia em outras $[\mathbf{1}, \mathbf{2}]$. Por isso, tais métodos são mais requisitados, justamente, na tentativa de minimizar os efeitos negativos causados devido ao atributo natural do sistema.

Dentre os pesquisadores dos sistemas TITO, Isermann [2] propôs um método que tem como princípio a escolha dos parâmetros do controlador de modo que esses valores mantenham o sistema a uma distância adequada do limite de estabilidade. As regras estipuladas foram estabelecidas para controladores analógicos ou controladores digitais, porém, com períodos de amostragem pequenos, tendo como valores de comparação as constantes de tempo do sistema.

$O$ pesquisador Alves [15] também dedicou-se na criação de métodos para sintonia de sistemas TITO, onde adotou-se como etapa inicial a avaliação quantitativa de um índice de desempenho chamado de integral do erro absoluto vezes o tempo (ITAE) nas saídas do processo e criou uma análise de 
ajustes refinados, baseado também, em uma região de estabilidade que deu a origem a uma tabela para o uso de correções futuras, mediante demanda do usuário.

Outro pesquisador responsável por pesquisas na área foi o Niederlinski [16], desenvolveu um método heurístico baseado na generalização do método de sintonia dos autores Ziegler e Nichols [17], para o caso de sistemas multivariáveis. $\mathrm{Na}$ implementação deste método, é necessária a substituição dos controladores por uma matriz de ganhos e realizar a identificação de pontos críticos e também das frequências críticas do processo.

\section{Modelo Utilizado}

O modelo utilizado por esse artigo é referente ao sistema multivariável composto por duas entradas e duas saídas, conhecido como TITO (Two Input Two Output), que pode ser observado na figura 1. É possível notar a interação mútua das malhas (cruzamento que interliga as matrizes do processo) que a tornam substancialmente mais complexas se comparado com os sistemas que possuem apenas uma entrada e uma saída - SISO (Single Input Single Output) $[\mathbf{1}, \mathbf{2}, \mathbf{1 3}, \mathbf{1 5}, \mathbf{1 6}]$ :

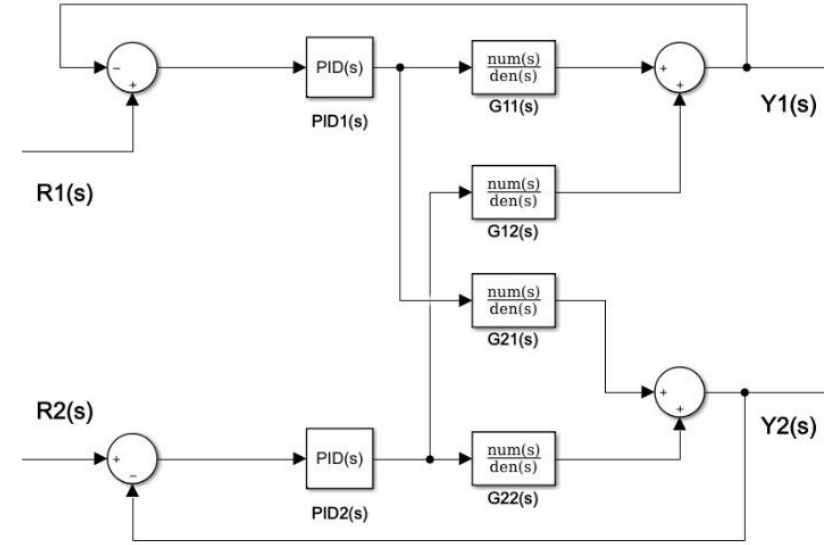

Figura 1: Sistema Multivariável 2x2 Fonte: O autor.

De acordo com a figura 1 , as entradas do processo correspondem a $\mathrm{R} 1(\mathrm{~s})$ e $\mathrm{R} 2(\mathrm{~s})$ e as saídas são expressas por $\mathrm{Y} 1(\mathrm{~s})$ e $\mathrm{Y} 2(\mathrm{~s})$. O controlador empregado foi o PID que, para essa configuração, foi utilizado de forma individual para o controle das duas malhas e pode ser descrito pelas seguintes equações [1]:

$$
\begin{aligned}
& P I D 1(s)=K p 1\left(1+\frac{1}{T i 1 s}+T d 1 s\right) \\
& P I D 2(s)=K p 2\left(1+\frac{1}{T i 2 s}+T d 2 s\right)
\end{aligned}
$$

Onde, Kp1 e Kp2 são os ganhos proporcionais, Ti1 e Ti2 são os tempos de integração e Td1 e Td2 são os tempos de derivação.

Os sistemas multivariáveis podem ser descritos por meio de uma matriz composta por funções de transferências $[\mathbf{2}, \mathbf{1 3}, \mathbf{1 5}, \mathbf{1 6}]$ :

$$
G(s)=\left[\begin{array}{ll}
G 11(s) & G 12(s) \\
G 21(s) & G 22(s)
\end{array}\right]
$$

$\mathrm{G}(\mathrm{s})$ é composto pelas matrizes que descrevem fisicamente 0 modelo do processo que será submetido ao ajuste.

\section{Descrição do Programa}

Nesse estudo o sistema foi modelado e simulado utilizando o "Simulink", uma extensão do programa MATLAB. A interface gráfica, GUI (Graphical User Interface), também é uma ferramenta disponível no MATLAB para criação de uma área de trabalho permitindo a comunicação entre a programação e o que será simulado. A Figura 2 demonstra como ficou o resultado final do programa.

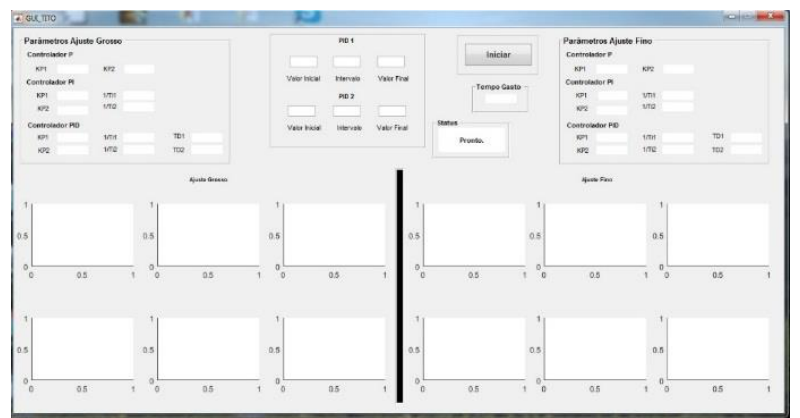

Figura 2: Interface (GUI) desenvolvido no MATLAB Fonte: O autor.

http://dx.doi.org/10.25286/repa.v4i1.956 
O programa foi criado para que qualquer usuário consiga executá-lo de forma simples, devido a isso, ele apresenta apenas um único botão, "Iniciar", capaz de executar toda metodologia que será discutida mais adiante. A única tarefa que o usuário deve executar é a de escolher o valor mínimo, o intervalo e o valor máximo para determinação de parâmetros importantes dentro da programação, conforme a Figura 3.

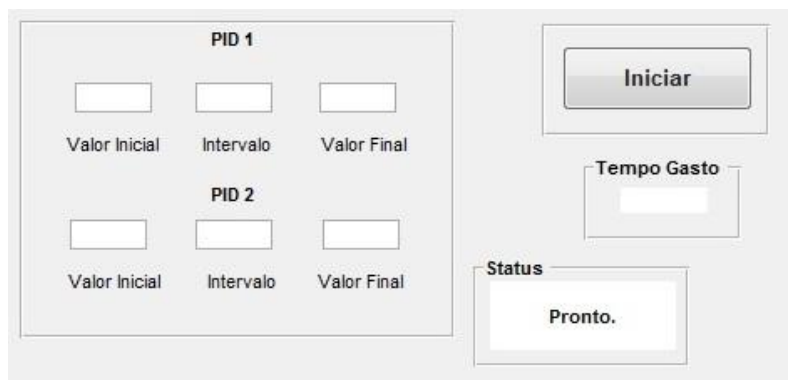

Figura 3: Slots, Botão Iniciar, Status e Tempo Gasto Fonte: O autor.

A entrada desses dados deve ser executada de acordo com a percepção do usuário, sendo que, quanto menor são os valores alocados, maior será o número de iterações, consequentemente, maior será o tempo gasto pelo programa para encontrar os ajustes adequados. A percepção referida nesse trabalho significa a escolha de um número arbitrário pelo usuário, entretanto, a seleção de um valor muito alto pode fazer com que o resultado divirja, levando o sistema à instabilidade. Vários trabalhos $[2, \mathbf{1 5}, \mathbf{1 6}, \mathbf{1 8}]$ explicam sobre a região de estabilidade que cada processo pode assumir, sendo alguns com valores elevados nos ganhos aplicados nos controladores e outros com valores bem mais sensíveis. Devido a essa condição individual que cada processo possui, recomenda-se que o usuário sempre adote um valor pequeno nos slots de valor inicial e intervalo para que a condição de ganho esteja nos limites aceitáveis garantindo assim um controle estável ao processo.

Os resultados que serão obtidos foram divididos em duas áreas distintas. A lateral esquerda corresponde ao primeiro nível de ajuste, também conhecido como ajuste grosso. Em contrapartida, o lado direito indica o nível mais refinado do ajuste, onde buscam-se correções que a primeira camada deixou de atuar. Se na parte superior são informados os dados quantitativos que serão utilizados nos controladores, na parte inferior ficam expostos os resultados visuais de cada ajuste, de ambas as saídas.

O programa é composto pelo algoritmo e o modelo usado na simulação, formando um ambiente gráfico executável, visto na Figura 2 . O sistema $2 \times 2$ demonstrado pela Figura 1, adquire uma nova forma, desta vez mais completo, composto por blocos que serão usados na simulação final, que pode ser visto na Figura 4.

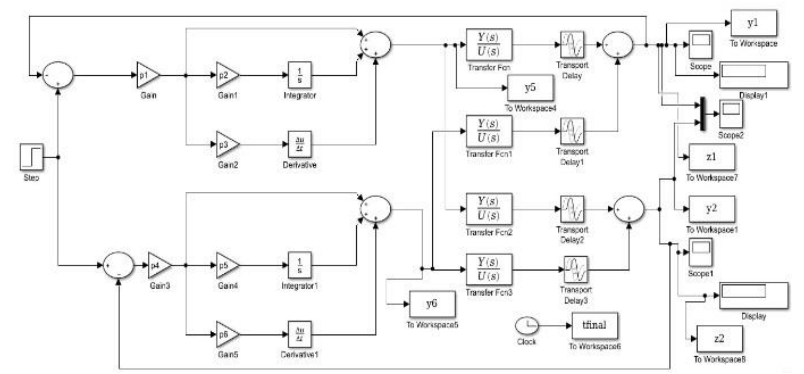

Figura 4: Arquivo Simulink do Sistema TITO Fonte: $O$ autor.

\subsection{Descrição da Metodologia Proposta}

Para a elaboração da metodologia proposta por este trabalho, utilizou-se inicialmente o conceito explorado pelos pesquisadores Ziegler e Nichols (ZN) [17]. Em 1942, os autores inventaram um método que, até nos dias atuais, é vastamente utilizado para sintonizar os controladores PID em sistemas monovariáveis. Para aplicação em sistemas TITO foram feitas modificações, a fim de conseguir obter resultados que satisfaçam os usuários, de modo que, as saídas mantenham-se estáveis.

O primeiro passo sugerido pelos pesquisadores ZN é levar o sistema a se tornar marginalmente estável, isto é, aplicar um ganho aleatório no controlador Proporcional, ignorando as outras ações (Integral e Derivativa), a fim de obter oscilações de mesma amplitude na saída. Nessa etapa seria possível coletar o ganho crítico e também o período de oscilação e por fim aplicar esses valores obtidos em uma tabela criada pelos próprios autores.

Com base nesse entendimento, foram feitas algumas modificações para atender a peculiaridade que os sistemas de duas entradas e duas saídas possuem. Inicialmente é necessário refrisar que em sistemas TITO, o controle é feito por dois 
controladores PID e que, diferentemente de sistemas SISO estes possuem ainda ligações cruzadas, onde uma pequena alteração em uma das entradas influencia diretamente sobre todo - sistema, tornando assim a tarefa mais desafiadora. Devido a esse motivo, aplicar um ganho onde levasse o sistema à beira da instabilidade não seria uma boa opção. É possível ver mais a fundo essa condição crítica e como influenciaria no sistema caso fosse adotado esse caminho, nos trabalhos dos pesquisadores Alves [15] e Anastácio [18].

Para contornar esse problema, foi construída uma nova maneira de obter os parâmetros iniciais de ganhos nos controladores. A proposta sugerida é trabalhar com o sobressinal, também conhecido como overshoot. O overshoot é o ponto mais elevado onde o sinal de saída ultrapassa o sinal de entrada. Por exemplo, quando aplicado um degrau unitário na entrada, espera-se um valor unitário na saída, qualquer outro valor que ultrapasse esse valor unitário e este for o ponto mais alto -se houver um conjunto de pontos-, denomina-se este ponto específico de overshoot. Com essa medida, de utilizar o valor de sobressinal, foi possível anular os efeitos causados caso fosse adotado o método dos pesquisadores ZN.

Para a implementação, foi necessário também estipular um valor numérico onde a saída atingisse esse sobressinal. Por meio de suscetíveis testes em diversos processos, foi possível determinar um número global que atendesse essa condição. O valor de sobressinal de referência proposto é o valor numérico 2 (dois). Sendo assim, para a metodologia proposta adota-se sempre a entrada como sendo um degrau unitário (step) nas entradas de ambos controladores e o valor de sobressinal que as saídas devem assumir sendo iguais à 2 . O valor do ganho obtido através da análise de overshoot recebeu a nomeação de "K" acompanhado da numeração "1" para indicar o primeiro controlador e " 2 " para indicar 0 segundo controlador.

Dando sequência, os autores ZN encontravam em sua metodologia na fase inicial dois parâmetros: o ganho crítico e o período de oscilação. O primeiro item obtido através do aumento gradativo do controle Proporcional até que se atingisse uma saída oscilatória. E o segundo item por consequência da primeira, obtinha uma saída oscilatória e então era possível coletar o período de oscilação desse sinal. No caso do período de oscilação é responsável para manutenção das outras ações que compõe o controle PID, o tempo de integração e o tempo derivativo. Inicialmente esses termos foram desprezados, mas com a obtenção do período, finaliza-se aplicando na mesma tabela que foi criada pelos autores $\mathrm{ZN}$, contemplando agora o restante das ações de controle. Para determinar os parâmetros das ações Integral e Derivativa, a metodologia proposta propõe utilizar uma função "findspeaks" presente no software Matlab. A função findspeaks determina os pontos máximos e mínimos dentro de um sinal gerado, e então fornece ao usuário o período de amostragem dos picos identificados. Aliado a programação desenvolvida, é coletado esses intervalos que servirá como parâmetro de ajustes nas ações remanescentes. Os períodos de amostragem coletados receberam o nome de "TT" acompanhado da numeração " 1 " para indicar o primeiro controlador e " 2 " para indicar o segundo controlador. Com a determinação desses parâmetros, encerra-se a fase de aquisição de dados necessários para fazer a sintonia inicial nos controladores PID.

\subsection{Descrição do Algoritmo}

O algoritmo proposto inicia-se com a obtenção dos dados designado pelo usuário. A primeira etapa consiste em identificar 0 valor de sobressinal (overshoot), em um valor estipulado em $100 \%$ do que é aplicado na entrada (setpoint), onde K1 é o valor do ganho de overshoot referente à primeira saída do controle proporcional, e $\mathrm{K} 2$ relativo ao valor de overshoot referente à segunda saída do controle proporcional.

Desse modo considera-se os outros parâmetros dentro do controlador PID, a ação integral e derivativa sendo nulas.

Outro ponto que deve ser destacado é a intercalação entre os ganhos K1 e K2, adota-se sempre $\mathrm{K} 1$ como sendo o primeiro ganho a ser encontrado, portanto, $\mathrm{K} 2$, assume inicialmente, um valor nulo.

Tanto R1(s) quanto R2(s), entradas do sistema, correspondem a um degrau unitário (step), onde o valor final e o tempo de mudança sempre serão unitários.

Dessa forma, o valor de sobressinal obtido, deve ser, referente ao momento em que a primeira saída atingir um valor numérico igual a 2 .

http://dx.doi.org/10.25286/repa.v4i1.956 
Sintetizando o primeiro procedimento, para atingir a condição em que $\mathrm{K} 1$ igual a 2, consideram-se os termos expressos nas equações 1 e 2, Kp2, Ti2, Td2, Ti1 e Td1 sendo nulos.

Dentro do algoritmo, o primeiro controlador proporcional, assume um ganho inicial referente ao valor inicial, variando até o valor final estipulado pelo usuário. O limite entre o valor inicial e o valor final é o valor de $\mathrm{Kp} 1$, já o ponto onde é determinado o valor de sobressinal da saída sendo igual a 2, refere-se ao termo K1.

$\mathrm{Na}$ segunda etapa o algoritmo irá realizar um procedimento similar. Encontra-se o valor de sobressinal de $\mathrm{K} 2$ onde ele atinja a condição da metodologia, de forma análoga, o usuário também estipulou o valor inicial, intervalo e valor final do segundo controlador, sendo assim, assume-se esse espaçamento como sendo os valores de $\mathrm{Kp2}$, no momento em que a segunda saída atingir um sobressinal igual a 2 , encontrase por fim, o termo K2.

Embora os termos Ti2, Td2, Ti1 e Td1 mantenham-se nulos, uma divergência ocorre nesse procedimento, o valor de $\mathrm{Kp} 1$ não é mais considerado nulo, $\mathrm{Kp} 1$ assume $\mathrm{o}$ valor correspondente a $\mathrm{K} 1$, encontrado na primeira etapa.

Sintetizando a segunda etapa do algoritmo, Kp1 é igual a K1, Ti2, Td2, Ti1 e Td1 são nulos.

O pesquisador Anastácio [18], demonstrou em seu trabalho que, em sistemas TITO, ao realizar o ajuste em um dos controladores, mesmo que todos os parâmetros do outro controlador sejam nulos, ainda assim é possível identificar uma forte interação de ambas saídas do sistema.

Diferentemente da metodologia dos notáveis pesquisadores Ziegler e Nichols [17], onde busca-se uma oscilação de mesma amplitude na saída em sua fase inicial, o algoritmo proposto, identificou que, em sistemas TITO ao tentar levar o sistema a se tornar marginalmente estável, cria-se uma dependência de múltiplas correções futuras.

Após o reconhecimento do sobressinal, o algoritmo identifica os pontos máximos da saída, formado por um vetor, por meio da função "findspeaks" presente no MATLAB. A observação desses pontos permite que, de forma autônoma,
- algoritmo consiga identificar o período, denominado aqui de TT1 e TT2 desse sinal, primeira e segunda saída, respectivamente. A Figura 5 é um fluxograma demonstrativo com as etapas percorridas pelo algoritmo.
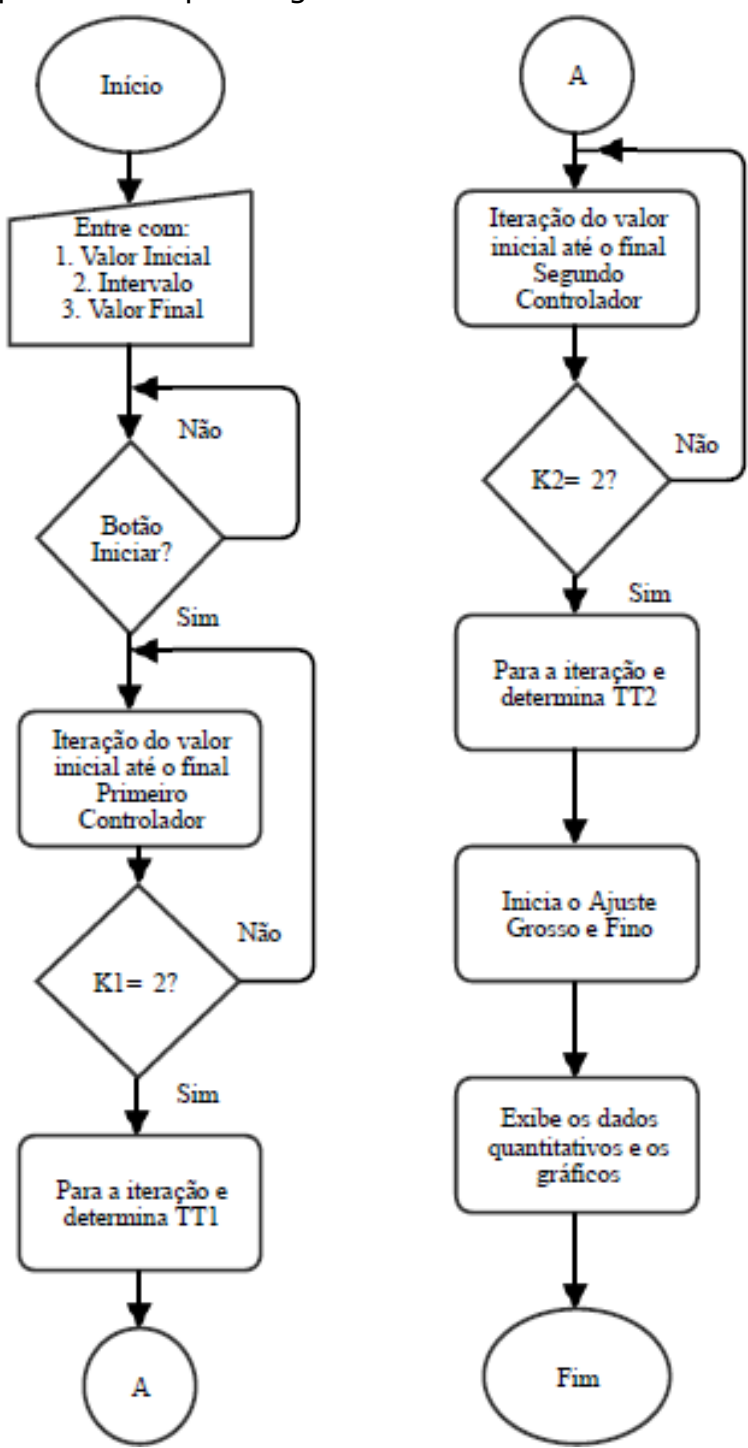

Figura 5: Fluxograma Geral do Algoritmo Proposto Arquivo Fonte: $\mathrm{O}$ autor.

\section{Ajustes}

O controlador PID, também conhecido como controlador de três termos, é composto por um termo proporcional para fechar a malha de realimentação, um termo integral para garantir erro nulo à referência e às entradas de perturbação, e o termo derivativo 
para melhorar a estabilidade e uma boa dinâmica [19]. Estão disponíveis diversas metodologias para sintonização dos controladores PID e a aplicação desse controlador é bem ampla, presente em setores como o automobilístico, metalúrgico, aeroespacial, entre outros [3].

O estudo do controlador PID é uma área bem ativa, conforme as recentes publicações $[\mathbf{2 0}, \mathbf{2 1}$, 22], e as pesquisas, de acordo com Padula [12], continuarão na tentativa de desenvolver novos métodos, bem como, a melhoria das técnicas já consolidadas, tentando assim, melhorar a eficácia operacional dos processos. Segundo ASTRöM e HÄGGLUND [7], os controladores PID continuarão sendo cada vez mais explorados devido a sua notória eficiência.

\subsection{Ajuste Grosso}

O procedimento subsequente é realizar o ajuste em sua primeira camada, conforme demonstrado pelo algoritmo que executa 0 método proposto. Para encontrar os primeiros parâmetros da camada do ajuste grosso, adotouse as equações presentes no trabalho de Ziegler e Nichols (ZN) [17], visto que, os índices iniciais de ajuste apresentam uma boa resposta tanto em sistemas SISO, como em sistemas TITO. Para o controle Proporcional foi adotada a seguinte expressão:

$$
P x=K x, \text { onde } x=1 \text { e } 2
$$

A próxima ação de controle é a Proporcional Integral (PI), sendo adotadas as equações:

$$
\begin{gathered}
P(P I) x=P x \times 0.45, \text { onde } x=1 \text { e } 2 \\
T i(P I) x=T T x \times 1.2, \text { onde } x=1 \text { e } 2
\end{gathered}
$$

E por fim, a ação completa, Proporcional Integral Derivativo (PID):

$$
\begin{gathered}
P(P I D) x=P x \times 0.6, \text { onde } x=1 \text { e } 2 \\
T i(P I D) x=T T x \times 0.5, \text { onde } x=1 \text { e } 2 \\
T d(P I D) x=T T x \times 0.125, \text { onde } x=1 \text { e } 2
\end{gathered}
$$

\subsection{Ajuste Fino}

Em muitas situações, apenas a execução do ajuste grosso na primeira esfera poderá não ser o suficiente para atender as expectativas do usuário [19]. Processos com comportamentos oscilatórios ou que apresentam lentidão na resposta podem perpetuar mesmo após a primeira camada de ajuste.

Devido a isso, o algoritmo executa um segundo nível de ajuste, na tentativa de obter melhores resultados. As equações utilizadas por esse trabalho para obtenção do ajuste fino foram construídas a partir de testes de controle realizadas em diversos processos de duas entradas e duas saídas, baseado numa avaliação quantitativa das respostas com aplicação de diferentes índices, sendo testadas nos mesmos processos utilizados para validar as metodologias dos autores Isermann [2], Alves [15] e Niederlinski [16]. Os índices utilizados para o ajuste fino variam de autor para autor, e como esse ajuste é bem particular, isto é, depende exclusivamente da percepção do usuário em avaliar se o estado do processo está de acordo com o esperado, foi sugerido um novo fator multiplicativo a partir de testes para ser aplicado nessa última etapa de ajuste, no controle proporcional, PI e PID, conforme demostram as expressões $10,11,12,13$, 14 e 15.

Para o ajuste fino no controlador Proporcional, ficou expresso:

$$
P x=K x \times 0.4, \text { onde } x=1 \text { e } 2
$$

Para a ação de controle PI, foi adotada a equação:

$$
P(P I) x=P x \times 0.6, \text { onde } x=1 \text { e } 2
$$

$T i(P I) x=T i(P i) x \times 0.9$, onde $x=1$ e 2

Finaliza-se com o ajuste fino no controlador PID: 


$$
\begin{gathered}
P(P I D) x=P(P I D) x \times 0.25, \text { onde } x=1 \text { e } 2 \\
T i(P I D) x=T i(P I D) x \times 0.9, \text { onde } x=1 \text { e } 2 \\
T d(P I D) x=T d(P I D) x \times 0.9, \text { onde } x=1 \text { e } 2
\end{gathered}
$$

\section{Resultados}

Para demonstrar o funcionamento do programa, juntamente com a método proposto, o mesmo foi aplicado no processo de uma incubadora neonatal [13]. A função de transferência obtida desse processo é definida como:

$$
G(s)=\left[\begin{array}{cc}
\frac{2.89 e^{-55 s}}{550 s+1} & \frac{-3.1 e^{-51 s}}{260 s+1} \\
\frac{-4.22 e^{-91 s}}{546 s+1} & \frac{22.22 e^{-16 s}}{180 s+1}
\end{array}\right]
$$

Como entrada, adotou-se um valor constante de temperatura de $30^{\circ} \mathrm{C}$ e para a variável umidade, houveram duas mudanças de referência, inicialmente em $t=2400$ s onde a umidade passa de $50 \%$ para $45 \%$ e por fim em $t=4800$ s com um acréscimo de $10 \%$ da umidade, subindo de $45 \%$ para 55\%, de acordo com a Figura 6.

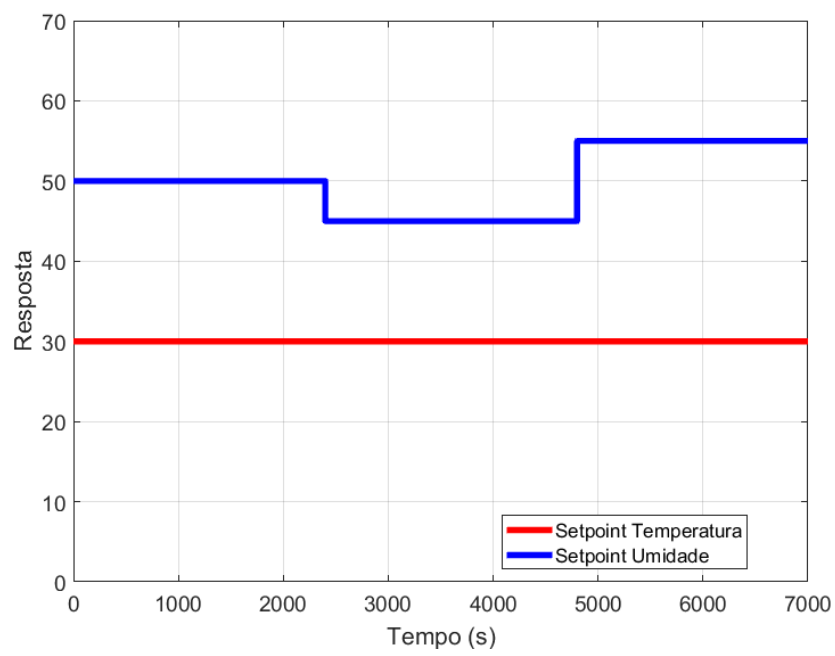

Figura 6: Setpoint R1(s) e R2(s) Fonte: $\mathrm{O}$ autor.
O programa foi executado e o tempo gasto para obter os parâmetros de ajuste, conforme demonstrado, foram de 15 segundos. Os parâmetros encontrados pelo programa podem ser vistos na tabela 1. Embora o método de sintonia dos pesquisadores $\mathrm{ZN}$ foram desenvolvidas para sistemas monovariáveis, os preceitos idealizados permitiram um novo caminho para o ajuste em sistemas multivariáveis. A Figura 7 demonstra com clareza os resultados visuais obtidos através do algoritmo proposto e o resultado aplicando o método Biggest Log-Module Tuning (BLT) [11]. O método BLT é uma técnica muito explorada pelos engenheiros de controle, embora seja de fácil implementação, necessita de diversas etapas para se alcançar uma sintonia adequada em sistemas TITO.

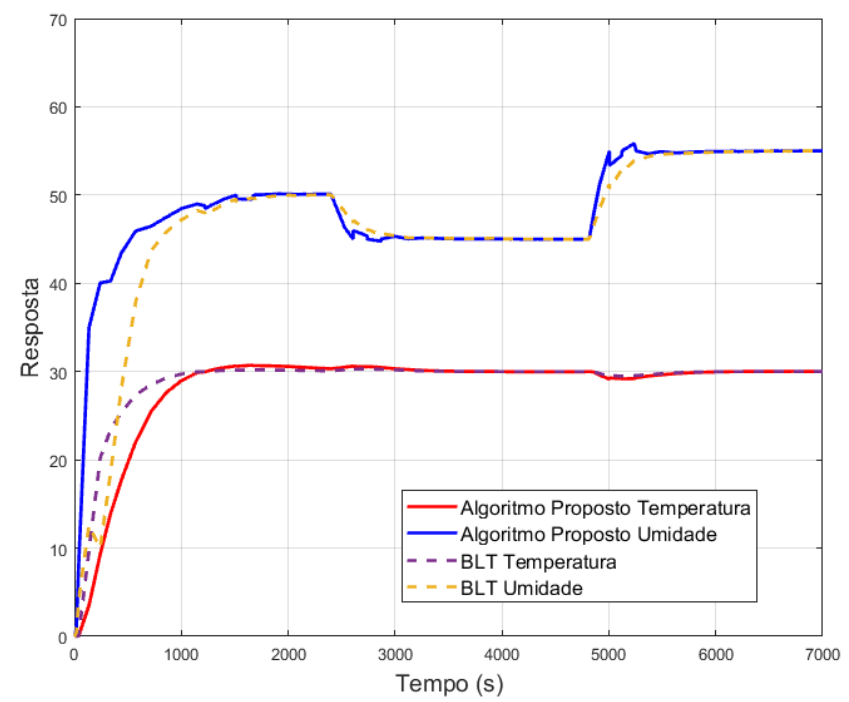

Figura 7: Resposta das Saídas Fonte: $\mathrm{O}$ autor.

Com os resultados demonstrados, é possível comprovar a eficiência do algoritmo proposto, conseguindo atingir os valores de referência com suavidade e sem oscilações bruscas. Ao comparar o algoritmo proposto com outra metodologia de controle consolidada, é possível notar que as respostas de ambas variáveis ficaram muito próximas, o que contribui para a validação do algoritmo.

Tabela 1: Parâmetros Encontrados Fonte: O autor.

\begin{tabular}{llll}
\hline Método & Kp & 1/Ti & Td \\
\hline
\end{tabular}




\begin{tabular}{cccc}
\hline BLT & 0.8633 & 0.0024098 & 0 \\
PID1(s) & & & \\
BLT & 0.0286 & 0.0082039 & 0 \\
PID2(s) & & \\
Proposto & 0.486 & 0.002718 & 0 \\
PID1(s) & & & \\
$\begin{array}{c}\text { Proposto } \\
\text { PID2(s) }\end{array}$ & 0.054 & 0.008748 & 0 \\
\hline
\end{tabular}

Para os valores encontrados pelo programa, foi definido um valor inicial de 0.1 , intervalo de 0.1 e valor final de 4, para ambos os controladores. 0 ajuste fino do controlador PI demonstrou ser o mais eficaz, por isso foi utilizado, dentre as opções disponíveis do programa proposto.

\section{Considerações Finais}

Nesse artigo foi proposto a criação de um programa com uma interface intuitiva capaz de fornecer um ajuste adequado em sistemas de duas entradas e duas saídas, em um curto espaço de tempo, sem que o usuário precise de qualquer conhecimento em sintonização de controladores. O algoritmo atendeu a lógica estabelecida para a solução da sintonia do controlador PID em sistemas TITO. Com os resultados obtidos, conclui-se que, o objetivo inicial foi alcançado e, além disso, a metodologia expressa fornece um novo método de ajuste nesses sistemas.

Resultados satisfatórios foram encontrados, conforme demonstrado pela resposta das saídas do processo escolhido para execução dos ajustes do algoritmo proposto. Vale ressaltar que, por se tratar do processo de uma incubadora neonatal, variações ríspidas nas variáveis envolvidas, não podem ser admitidas, podendo comprometer a vida do recém-nascido. E o algoritmo proposto conseguiu atender essa característica de vital importância.

$\mathrm{O}$ algoritmo também pode ser implementado como forma inicial de ajuste em qualquer sistema TITO, para poder pôr em prática o funcionamento imediato do processo, e então, o operador, futuramente poderá implementar um controle mais particular para atender a demanda individual do processo a ser controlado. Essa possibilidade atende tanto $o$ operador que possui 0 conhecimento de sintonia, que no caso pode utilizar o algoritmo para comparar os resultados com o método adotado por ele ou pelo operador que desconhece de sintonia, mas precisa pôr o processo em funcionamento para não comprometer a linha produção, por exemplo.

Trabalhos futuros podem ser desenvolvidos com a intenção de obter o modelo de uma planta real e criar uma comunicação online entre o processo e os ajustes. Além disso, é possível criar dentro do programa alternativas de sintonia baseado nas experiências do usuário, bem como, a construção de pacotes com outras metodologias consolidadas.

\section{Referências}

[1] SKOGESTAD, S.; POSTLEWAITE, I. Multivariable Feedback Control: Analysis and Design, $2^{a}$ Ed. John Wiley \& Sons, New York, cap. 3 e 4, pp. 69 - 168, 2005.

[2] ISERMANN, R. Digital Control Systems. German: Springer Verlag, $1^{\mathrm{a}}$ Ed, p. 316 $351,1981$.

[3] RUBAAI, A., SITIRICHE, M. J. C.; OFOLI, A. $R$. Design and implementation of parallel fuzzy pid controller for highperformance brushless motor drives: An integrated environment for rapid control prototyping. IEEE Transactions on Industrial Electronics, p. 1090 - 1098, 2008. https://ieeexplore.ieee.org/document/45788 08/

[4] CONG, S.; LIANG, Y. Pid-like neural network nonlinear adaptive control for uncertain multivariable motion control systems. IEEE Transactions on Industrial Electronics, p. 3872 - 3879, 2009. https://ieeexplore.ieee.org/document/48120 95/

[5] CHENG, C. Y. Autotuning of PID controllers: A Relay Feedback Approach. Springer-Verlag, London, 2a Ed., 2009.

[6] ARRUDA, L., SWIECH, M., JUNIOR, F.; DELGADO, M. Um método evolucionário para sintonia de controladores PI/PID em processos multivariáveis. Revista Controle e Automação, p. 1 - 16, 2008. 
http://www.scielo.br/scielo.php?script=sci _arttext\&pid=S0103-

17592008000100001

ASTRÖM, K.; HÄGGLUND, T. PID

Controllers: Theory, Design and Tuning. Instrument Society of America, 2a Ed, cap. 4, p. 120 - 199, 1995.

[8] NEMA, S.; PANDHY, P. K. CO PID Controller for TITO Processes. IEEE International Conference on Intelligent Systems and Control (ISCO), p. 608 612, 2015.

https://ieeexplore.ieee.org/document/728 2339/

[9] LI, X., WU, R.; ZHANG, W. The IMC-PID Controller Design for TITO Process Using Closed-loop Identification Method. IEEE International Conference on Control, Automation, Robotics \& Vision (ICARCV), p. 1339 - 1344, 2014. https://ieeexplore.ieee.org/document/706 4510/

[10] PAUL, P. K., DEY. C.; MUDI, R. K. Design of Multi-loop IMC-PID Controller for TITO Process with Dead Time. IEEE International Conference on Control, Instrumentation, Energy \&

Communication, p. 45 - 49, 2016. https://ieeexplore.ieee.org/document/751 3796/

[11] LUBYEN, W. L. Simple Method for Tuning SISO Controllers in Multivariable Systems. Ind. Eng. Chem. Process Des. Dev, vol. 25, p. 654 - 660, 1986.

https://pubs.acs.org/doi/abs/10.1021/i20 0034a010

[12] PADULA, F.; VISIOLI, F. Tuning rules for optimal and fractional-order PID controller, Journal of Process Control, $v$. 21, n. 1, p. 69-81, 2011. https://www.sciencedirect.com/science/... /S0959152410001927
[13] NETO, J. O. B. Controle Multivariável de Temperatura e Umidade Aplicado em Incubadoras Neonatais. Dissertação de Mestrado, Universidade Federal do Ceará, 2010.

[14] RIBEIRO, J. M. S., SANTOS, M. F.; SILVA, M. $F$. Comparison of PID controller tuning methods: analytical/classical techniques versus optimization algorithms. 18th International Carpathian Control Conference (ICCC), p. 533 - 538, 2017.

https://ieeexplore.ieee.org/document/79704 $58 /$

[15] ALVES. P. G. Controle PID Multivariável: Simulações e Procedimento de Ajuste. Monografia de Graduação, Universidade Federal do Rio de Janeiro, 1988.

[16] NIEDERLINSKI, A. A heuristic approach to the design of linear multivariable interacting control systems. Automatica, v. 7, p. $691-701,1971$.

https://www.sciencedirect.com/science/articl e/pii/0005109871900070

[17] ZIEGLER, J.; NICHOLS, B. Optimum settings for automatic controls. Transactions ASME, Vol. 64, p. 759 - 768, 1942.

https://staff.guilan.ac.ir/staff/users/chaibakh sh/fckeditor_repo/file/documents/Optimum $\%$ 20Settings\%20for\%20Automatic\%20Controll ers\%20(Ziegler\%20and\%20Nichols, \%20194 2).pdf

[18] ANASTÁCIO, C. M. S. Revisão de uma Metodologia de Empírica de Ajuste do Controle PID para Sistemas TITO Usando o Matlab. Monografia de Graduação, Centro Universitário de Volta Redonda, 2017.

[19] FRANKLIN, G. F., POWELL, J. D.; NAEINI, A. E. Sistemas de Controle para Engenharia. Bookman Editora, 6a Ed, p. 167 - 177, 2013. 
[20] BADGWELL, T. Robust Model Predictive Control of Stable Linear Systems, International Journal of Control, v. 68, p. $797-818,1997$.

https://www.tandfonline.com/doi/abs/10. ../002071797223343

[21] SEFIANI, N., CHEBLI, S.; ELAKKARY, A. PID controller tuning using multiobjective ant colony optimization applied to TCP/AQM networks. IEEE Automation and Computing (ICAC), 2017. https://ieeexplore.ieee.org/document/808 2041/

[22] SHUKOR, N. S. A., AHMAD, M. A.; TUMARI, M. Z. M. Data-driven PID tuning based on safe experimentation dynamics for control of liquid slosh. IEEE Control and System Graduate Research Colloquium (ICSGRC), p. 62 66, 2017.

https://ieeexplore.ieee.org/document/807 0569/ 